\title{
Cómo mejorar las tasas de retención en los tratamientos de drogodependencias
}

\author{
Secades Villa, R.; Fernández Hermida, J.R.
}

Profesores Titulares del Departamento de Psicología. Universidad de Oviedo.

\section{RESUMEN}

La alta tasa de abandonos de los programas de tratamiento es uno de los problemas más graves en el ámbito de las drogodependencias. En la mayoría de las ocasiones, el abandono de un paciente significa la recaída en el consumo de drogas y el empeoramiento de otras áreas del estilo de vida de éste. En este artículo se revisan las tasas de abandono, los factores relacionados con la retención y, sobre todo, las estrategias de intervención para incrementar los niveles de retención en los programas de tratamiento. Se proponen tres tipos de estrategias: las relacionadas con los pacientes, con los parámetros del tratamiento y las que tienen que ver con los terapeutas. Los estudios empíricos han demostrado que estos procedimientos pueden ser muy eficaces a la hora de reducir las tasas de abandono y, por tanto, de mejorar los resultados de la intervención independientemente de la modalidad del programa.

Palabras clave: Abandono, retención, estrategias de intervención.

\section{SUMMARY}

The high rate of dropouts from treatment programs is one of the most serious problems in the substance abuse field. In most cases, a patient's dropout brings about a relapse and into drug consumption and worsening of his/her life style conditions. This article reviews the rates of dropouts, the factors related with retention, and, mainly, intervention strategies to increase levels of adherence in the treatment programs. We propose three types of strategies: those related to the patient, those related to the parameters of treatment, and those related to the therapist. Empirical studies have demostrated that these procedures may be very effective to reduce the rates of droputs and, therefore, to improve treatment outcome in all tipes of programs.

Key words: Dropout, retention, intervention strategies.

\section{INTRODUCCIÓN}

$\mathbf{L}$ a falta de adhesión a los tratamientos es una de las principales causas de fracaso terapéutico y es un problema con el que se enfrentan todos los profesionales de la salud. Se trata de un fenómeno que afecta a todo tipo de pacientes, sea cual sea su dolencia y su condición. Sin duda, una de las formas más graves de falta de adhesión es el abandono prematuro del tratamiento.

En el ámbito de las drogodependencias este fenómeno se presenta con una altísima frecuencia. Ya en el año 1972, O'Malley, Anderson y Lazare resaltaron que sólo el $3 \%$ de los heroinómanos que acudían a una clínica psiquiátrica ambulatoria volvían a la segunda visita que se les había aconsejado.
En el tratamiento de la adicción a las drogas (cualquiera que ésta sea) se ha podido observar una tasa de abandonos muy elevada, siendo dicho abandono casi un pre-requisito de la vuelta al consumo. Este hecho justifica conceder una atención especial a este grave problema de los tratamientos de consumo de drogas.

Antes que nada, cabría realizar unas matizaciones de la definición del abandono, ya que el mismo concepto parece incluir casuísticas distintas, desde pacientes que fallan en volver a citas programadas, a otros que expresamente rechazan el tratamiento propuesto o aquellos que son expulsados por falta de cooperación o carencia de respuesta terapéutica.

Resulta útil aquí la clasificación propuesta por Des Jarlais, Joseph y Schmeidler (1983) que divide la salida de un programa de tratamiento de drogodepen- 
dencias en tres categorías: la expulsión, que se produce cuando el paciente es despedido por violar las reglas del programa; el abandono prematuro, que hace referencia al hecho de que el paciente deje voluntariamente el tratamiento y el equipo cree que es necesario que siga en el mismo; y el tratamiento completo (o alta terapéutica), que se produce por decisión del equipo terapéutico, ya que ha alcanzado los objetivos que se habían propuesto.

Según Craig y Olson (1988), existe abandono cuando el paciente no completa el tiempo de permanencia planeado. Berger y Smith (1978) lo definen como una petición por parte del paciente de suspender el tratamiento en contra de la consideración del equipo, normalmente por un rechazo hacia la reglamentación del centro.

En definitiva, la definición del concepto de abandono terapéutico hace referencia a la interrupción de un determinado tratamiento antes del tiempo programado y por decisión del paciente.

\section{TASAS DE ABANDONO YIO RETENCIÓN EN LOS PROGRAMAS DE DROGODEPENDENCIAS}

En varios estudios se han encontrado tasas de abandonos muy superiores en los tratamientos de drogodependencia en comparación con otro tipo de problemas (por ejemplo, Swett y Noones, 1989; MacNair y Corazzini, 1994; Wierzbicki y Pekarik, 1993).

En una revisión clásica realizada por Baekeland y Lundwall (1975) se encontró una tasa de abandono del $64 \%$ a los 12 meses en los Programa de Metadona y de $88 \%$ a los 3 meses en los Programas Libres de Drogas.

En la Tabla 1 se muestra las tasas de abandono recogidas de algunos de los estudios revisados. Se trata de datos difíciles de comparar por tratarse de diferentes tipos de programas y porque son distintos periodos de tiempo los que se tienen en cuenta. No obstante, una conclusión general que se puede extraer es que la mayoría de los estudios de seguimiento sobre programas dirigidos a drogodependientes refieren tasas de abandono muy elevadas, sobre todo cuando se trata de programas libres de drogas.

Tabla 1. Tasas de abandono en los programas de drogodependencias

\begin{tabular}{lccc}
\hline Estudio & Tipo de programa & Tasas de abandono (\%) & Seguimiento \\
\hline Simpson (1984) DARP & MM & 64 & \\
& CT & 71 & 10 meses \\
\hline Hubbard et al. (1984) TOPS & PAL & 68 & $3-6$ meses \\
& DA & $35-50$ & $1-3$ meses \\
\hline De Leon y Schwartz (1984) & PALD & $25-44$ & $1-3$ meses \\
\hline Sánchez-Carbonell et al. (1988) & CT & $36-60$ & 1 año \\
& PALD & 78 & 1 mes \\
& PALD & 91 & $1-3$ meses \\
\hline Ochoa et al. (1992) & & 93 & $3-6$ meses \\
\hline Machado y Girón (1993) & & 71 & $6-12$ meses \\
\hline Gutierrez et al. (1995) & NAL & 69 & 12 meses \\
\hline Del Rio et al.(1997) & PALD & 84 & 6 meses \\
\hline Secades y Menéndez (1997) & NAL & 86 & 3 meses \\
\hline Secades et al. (1998) & MM & 90 & 6 meses \\
\hline D'lppoliti et al. (1998) & PALD & 53 & 12 meses \\
\hline Aszalos et al. (1999) & CT + PALD & 72,5 & 6 meses \\
\hline MM: Programa de Mantenimiento con Metadona. CT: Comunidad Terapéutica. PALD: Programa Ambulatorio Libre de Drogas. NAL: Programa con naltrexona \\
\hline
\end{tabular}




\section{EL SIGNIFICADO DE LA RETENCIÓN EN LOS PRO- GRAMAS DE DROGODEPENDENCIAS}

¿Qué importancia o qué repercusión tiene este fenómeno en el ámbito de las drogodependencias?

Así como en determinados trastornos psicopatológicos, el abandono no significa necesariamente una falta de mejoría o un empeoramiento del estado del paciente, el panorama es distinto en el alcoholismo o la adicción a la heroína. En estos ámbitos, la mayoría de los abandonos suponen un mal pronóstico, sobre todo si éstos se producen en los primeros momentos del tratamiento. Es decir, la mayoría de los pacientes que abandonan recaen.

Numerosos estudios han obtenido resultados que relacionan el abandono prematuro del tratamiento en drogodependencias con una mayor probabilidad de recaída, y el mantenimiento en el mismo con un mejor pronóstico o con mejores resultados. Por tanto,

la eficacia de un tratamiento de drogadicción está altamente correlacionada con la retención.

Uno de los primeros autores que llamó la atención sobre esta relación fue Vaillant (1966), quien sugirió la existencia de una relación inversa entre la duración de la estancia de los pacientes en el Hospital Lexington de Nueva York y la recaída posterior.

Estudios sucesivos han confirmado esta hipótesis y han sugerido que un indicador de buen pronóstico es la permanencia en el programa por encima de los tres meses (Sirotnik y Roffe, 1978; Simpson, 1984).

Por ejemplo, datos extraídos del DARP (Simpson, 1984) confirmaron que el resultado era más favorable a medida que se incrementaba la duración del tratamiento durante el primer año y que los pacientes que se mantenían en tratamiento menos de 3 meses no presentaron mejores resultados que los que llevaron a cabo simplemente una desintoxicación ambulatoria o los que recibieron una atención inespecífica en un grupo de admisión al programa.

Además, la importancia de la retención en el resultado del tratamiento ha sido confirmada en varios tipos de sustancias. Por ejemplo, Wells, Peterson y Gaine (1994) comprobaron que la retención predecía la reducción del consumo en diferentes tipos de programas de tratamiento (externos y residenciales) de adicción a la cocaína.

Por otro lado, el tipo de salida del programa también se relaciona con el efecto que ejerce éste sobre la conducta de los sujetos a largo plazo. Las revisiones de Tucker, Vuchinich y Harris (1985) y de Stark (1992) concluyen de una forma muy clara que los sujetos que terminan los tratamientos de abuso de alcohol y otras drogas tienen muchos mejores resultados que aquellos que abandonaban.
De lo expuesto anteriormente podemos concluir que, tanto el tiempo de permanencia del sujeto como el tipo de finalización del mismo son dos factores que influyen poderosamente en la eficacia de los programas. Mantenerse en el tratamiento y terminarlo tiene un potente valor predictivo sobre los resultados. Incluso, en algunos programas, por ejemplo los de mantenimiento con metadona, la retención es el criterio fundamental para medir la eficacia del tratamiento.

\section{FACTORES RELACIONADOS CON EL ABANDONO DEL TRATAMIENTO}

Dada la importancia del abandono, una línea de investigación de gran interés es la de conocer los factores relacionados con este fenómeno.

El estudio de los factores determinantes del abandono es decisivo a la hora de adecuar los tratamientos a las circunstancias de cada paciente o de derivarlo al programa que más se adapte a sus características. De la misma manera, el conocimiento de dichas variables permitiría mejorar las tasas de retención en los distintos programas mediante la aplicación de técnicas específicas orientadas a este fin, con el consiguiente aumento de la eficacia de los mismos.

Las variables relacionadas con la adhesión de los drogodependientes a los programas de tratamiento son muchas y diversas. La mayoría de los trabajos suelen distinguir entre variables de los pacientes y las variables referidas al tratamiento (procesos o parámetros del tratamiento) (Tabla 2).

Entre las variables individuales se pueden distinguir, a su vez, tres tipos: variables sociodemográficas, variables de "estado" (que se refieren al perfil general de gravedad) y variables "dinámicas," que se corresponderían con los aspectos disposicionales del paciente, tales como la motivación hacia el cambio y la actitud general ante el tratamiento.

También se habla, dentro de las variables individuales, de las variables fijas, que incluyen las demográficas y los antecedentes (de la historia del individuo) y las variables dinámicas que se refieren a las circunstancias y la motivación bajo las cuales los pacientes ingresan en el programa de tratamiento.

Los primeros trabajos que se interesaron por estas cuestiones fueron realizados a mediados y finales de los años setenta. En concreto, tres de los estudios pioneros fueron los de Aron y Daily (1976), Wexler y De Leon (1977) y Gossop (1978), los dos primeros realizados con pacientes ingresados en un programa residencial, en concreto en una Comunidad Terapéutica, y el último en el Maudsley Hospital de Londres, un tratamiento de tipo ambulatorio. 
Tabla 2. Factores relacionados con el abandono terapéutico

\begin{tabular}{ll}
\hline Referidas a los sujetos & Referidas al tratamiento \\
\hline -Sociodemográficas & -Recursos del programa \\
-Personalidad & -Orientación teórica \\
-Psicopatología & -Relación terapéutica \\
-Historia de consumo (severidad de la adicción) & -Características del terapeuta \\
-Situación legal & -Experiencia y composición del equipo terapéutico \\
-Salud & -Estabilidad de los miembros del equipo \\
-Motivación para el tratamiento & -Apoyo familiar \\
& -Contexto terapéutico (residencial/ambulatorio) \\
& -Tratamiento farmacológico/psicológico \\
& -Tipo de fármaco y dosis
\end{tabular}

Desde entonces, han sido muchos los trabajos publicados que indagan acerca de las variables relacionadas con la retención de los pacientes en los programas de drogodependencias, aunque no todos ellos llegan a conclusiones coincidentes.

En la tabla 2 se observan las variables más habitualmente estudiadas, organizadas en dos categorías: variables relacionadas con los pacientes y variables relacionadas con los programas.

En cuanto al primer grupo, se trataría de responder a las preguntas: ¿quién abandona los tratamientos?, o bien, ¿son los sujetos que permanecen diferentes a los que abandonan prematuramente?

Las investigaciones que tratan de buscar características de los pacientes asociadas a la retención han obtenido resultados dispares y, en general, estas variables suelen contribuir poco a la varianza de la retención.

Los resultados de estos estudios parecen sugerir que los sujetos que abandonan tienen una adicción más severa que los que permanecen y finalizan los tratamientos. Esta severidad de la adicción se podría operativizar atendiendo a variables como: mayor probabilidad de ser policonsumidor, psicopatología asociada, con menos apoyo familiar, con mayor número de años consumiendo drogas, con antecedentes criminales y pobre motivación hacia el tratamiento.

De la misma manera, podríamos afirmar que existiría otro grupo de variables, como las variables sociodemográficas (como la edad o el sexo) y los factores de personalidad, que parecen no estar relacionadas con el abandono de los programas para drogodependientes.

En este sentido, la conclusión que se extrae con respecto a los programas para drogodependientes es muy similar a las que Garfield (1994) extrajo en su revisión de los tratamientos psicológicos en general. En esta revisión, el autor concluye que las variables que tienen que ver con la clase social están inconsistentemente relacionadas con el abandono, mientras que variables como la edad, sexo y las variables que miden los tests psicológicos no han tenido demasiado éxito a la hora de predecir el abandono o la finalización de los tratamientos psicológicos.

En lo que respecta a los parámetros del tratamiento, han sido también muchas y muy diferentes las variables estudiadas. En general, se podría afirmar que existe mayor retención cuanto mayor sea la estabilidad de los terapeutas y si existe un alto grado de implicación de la familia. Además, las tasas de retención son más altas en los programas residenciales frente a los ambulatorios, en los tratamientos farmacológicos frente a los psicológicos y con dosis altas frente a más bajas del mismo fármaco. Por tanto, el ranking de la retención de los programas sería, por este orden: (1) tratamiento con metadona (y en general con agonistas), (2) programas residenciales y (3) programas ambulatorios.

\section{ESTRATEGIAS DE INTERVENCIÓN PARA EL INCREMENTO DE LA RETENCIÓN}

En los últimos años ha emergido con fuerza una nueva línea de trabajo que trata de buscar estrategias para incrementar la retención de los pacientes en los programas de tratamiento, sobre todo, en los programas psicosociales, en donde las tasas de abandono son mucho más elevadas que en los tratamientos farmacológicos.

En general, la mayoría de estos estudios parten de tres asunciones sobre la retención (Carroll, 1997):

-La retención tiene que ver con la adaptación o el ajuste entre el paciente, el contexto, el clínico y el tratamiento.

Tradicionalmente, las variaciones en la retención (y en los resultados) han sido atribuidas a determinadas características de los pacientes. Quizás, un punto de vista más acertado es que la retención tienen más que ver con lo que los clínicos hacen en los progra- 
mas de tratamiento que con las características de los pacientes. Y sobre todo, con la relación entre ambos factores.

-En la mayoría de las ocasiones, los abandonos ocurren en las primeras fases del tratamiento.

Del mismo modo que existe poca consistencia sobre las características de los pacientes que abandonan, existe una fuerte evidencia de que la mayoría de los abandonos ocurren en su mayoría en las primeras fases de tratamiento (Baekelend y Lundwall, 1975; De Leon, 1991; Kooyman, 1993/1996; Secades, Fernández Rodríguez y Fernández Hermida, 1998) y de que estos abandonos son, además, los más graves (los que tienen peor pronóstico).

La tasa más alta de abandonos se da en los primeros 30 días, continúa creciendo hasta los 3 meses y se empieza a reducir poco a poco a partir de ese momento. La probabilidad de una permanencia continuada en el programa aumenta considerablemente después de 90 días. Esto es, la probabilidad de abandono decrece con el paso del tiempo.

Así, la representación gráfica de las curvas de retención da lugar a un patrón característico en forma de hipérbola (Secades, Fernández Rodríguez y Fernández Hermida, 1998), que es el mismo para todos los programas (sobre todo los libres de drogas), aunque difieren en las tasas absolutas de retención.

Por tanto, se hace necesario recalcar la importancia que tiene la implementación de estrategias específicas con el fin de mejorar el ajuste de los pacientes en las fases tempranas de máxima vulnerabilidad.

\section{-La retención es un criterio del resultado del tratamiento.}

Una mejor retención tiende a estar asociada con mejores resultados, en términos de reducción de consumo de drogas y de mejora en otras variables del estilo de vida de los sujetos. Por extensión, un criterio de eficacia de los tratamientos del abuso de drogas es su capacidad para retener a los pacientes.

Se han probado varias estrategias para reducir las tasas de abandono de los programas. Desde nuestro punto de vista, estos procedimientos se podrían agrupar en tres categorías (Tabla 3): (1) estrategias relacionadas con los pacientes, (2) estrategias relacionadas con los programas y (3) estrategias relacionadas con los terapeutas.

\section{Tabla 3. Estrategias para el incremento de la retención}

\section{Referidas a los pacientes}

(1) Adaptación de los pacientes a los programas de tratamiento: restringir los criterios de inclusión

(2) Incrementar la motivación

\section{Referidas a los programas}

(3) Incrementar la dificultad para entrar en el programa de tratamiento

(4) Anticipar la heterogeneidad: adecuar los programas a las condiciones y necesidades de los sujetos

(5) Implicar a las personas allegadas en el tratamiento

(6) Terapias de incentivo (manejo de contingencias)

(7) Combinar terapias farmacológicas con psicológicas

\section{Referidas a los terapeutas}

(8) Formar

(9) Incentivar

(10) Procurar estabilidad y continuidad

\section{(1) Estrategias relacionadas con los pacientes}

\section{a) Adaptación de los pacientes a los programas de tratamiento}

Se trataría de restringir la variabilidad de los sujetos. Es decir, de restringir los criterios de inclusión/exclusión, de tal manera que se seleccionasen aquellos que, por sus características, se adapten mejor a las condiciones del programa y, por tanto, se incremente la probabilidad de finalizar el mismo y de obtener un resultado satisfactorio.

Los estudios sobre la adecuación paciente-tratamiento parten del hecho de que los tratamientos no funcionan igual en todos los sujetos. Este tipo de investigaciones intentan buscar la relación idónea entre las características de los pacientes y los distintos modelos de tratamiento. Es decir, se plantean la cuestión de qué programas de tratamiento para la drogadicción funcionan mejor con qué tipos de pacientes.

Así, existe un consenso, más o menos generalizado, de los perfiles idóneos de los pacientes para cada una de las modalidades de tratamiento existentes. En el trabajo de Sánchez y Matellanes (1993) se hace un breve repaso de los recursos asistenciales en drogodependencias y se define el perfil de los sujetos que se adaptaría mejor a cada uno de éstos. 
Desde nuestro punto de vista, el criterio de adecuación de los pacientes a las diferentes opciones terapéuticas existentes debería ser todavía menos rígido. Lo idóneo sería una flexibilización mucho más amplia de los programas. Es decir, una prescripción individualizada del tratamiento. Así, se podría aplicar un apoyo farmacológico dentro de un programa psicosocial. Por ejemplo, un individuo podría estar ingresado en una Comunidad Terapéutica y, al mismo tiempo, estar tomando metadona o naltrexona cuando tuviera salidas al exterior. En la actualidad, la oferta de alternativas terapéuticas continúa siendo demasiado rígida $y$, en determinados contextos, casi es un anatema pensar en la posibilidad de ofrecer a los pacientes una combinación de diferentes opciones que, hoy por hoy, se encuentran en la práctica muy alejadas.

\section{b) Incremento de la motivación}

Existen muchos trabajos en donde la motivación para el tratamiento ha resultado ser una variable importante para la retención en diferentes tipos de programas (por ejemplo, Gossop, 1978; Erickson, Stevens, McKnight y Figueredo,1995; Simpson, Joe y Rowan-Szal, 1997; Joe, Simpson y Broome, 1998).

De Leon junto con otros colaboradores ha desarrollado la escala CMRS para la evaluación de esta variable (De Leon y Jainchill, 1986; De Leon, Melnick,, Kressel y Jainchill, 1994). Varios trabajos han encontrado que la puntuación en este cuestionario es un buen predictor de la retención en los programas de tratamiento, sobre todo a corto plazo (De Leon, Melnick y Kressel, 1997; Melnick, De Leon, Hawke, Jainchill y Kressler, 1997; González-Saiz et al., 1998).

En cuanto a las estrategias para incrementar la motivación, se hace necesario mencionar el modelo de intervención denominado "Entrevista Motivacional" de Miller y Rollnick (1991). Se trata de un paquete de técnicas de motivación que han de llevarse a cabo en los momentos iniciales del tratamiento, por tratarse de la fase en donde se producen la mayoría de los abandonos.

La entrevista Motivacional ha sido aplicada con éxito con todo tipo de adictos, por ejemplo, con alcohólicos (Bien, Miller y Tonigan, 1993) y con heroinómanos (Saunders, Wilkinson y Allsop, 1991).

El popular Modelo Transteórico (Prochaska y DiClemente, 1986; Prochaska, DiClemente y Norcross, 1992) podría también entenderse, al menos en parte, como otro intento de definir la motivación. De acuerdo con sus autores, la mayoría de los adictos que acuden a un programa están en el estadio de precontemplación, mientras que pocos tratamientos incluyen estrategias orientadas hacia este estadio. Se trataría de incluir los procedimientos (procesos) de cambio para mover el sujeto desde la precontemplación a estadios más avanzados, en las primeras fases del tratamiento. Entre otros: identificar el estadio de cambio del sujeto, centrarse en los intereses y preocupaciones inmediatos, llevar a cabo un "balance decisional" y realizar una confrontación objetiva de las consecuencias personales del abuso de drogas.

\section{(2) Estrategias relacionadas con los programas: modificación de los parámetros del trata- miento}

\section{a) Incrementar la dificultad para entrar en el pro- grama de tratamiento}

Un procedimiento para reducir los abandonos consiste en dificultar la entrada de los sujetos en los programas de tratamiento. Aunque no se suele hacer con esta intención muchos programas utilizan de forma rutinaria esta estrategia.

Se ha comprobado que retener a los sujetos en una lista de espera, mientras que, al mismo tiempo, llevan a cabo determinadas prescripciones antes del tratamiento como condición de entrada en el mismo (por ejemplo, autorregistros, cuestionarios o revisiones médicas) mejora las tasas de retención.

Algunos estudios bien controlados han estudiado el efecto que la rapidez del ingreso en el programa de tratamiento tiene sobre la retención. En el estudio de Stark, Campbell, y Brinkerhoff (1990), los sujetos (adictos a diferentes tipos de sustancias) fueron asignados aleatoriamente a dos condiciones experimentales: en el primer grupo, los pacientes entraban en la clínica inmediatamente después de solicitar el ingreso. Los sujetos del segundo grupo ingresaban en el centro por el procedimiento habitual (con una media de 9,7 días tras el primer contacto). Los resultados mostraron que las tasas de abandono eran significativamente más altas en el primer grupo, lo que sugiere que la atención rápida de las demandas de tratamiento puede tener efectos negativos sobre los resultados posteriores del tratamiento.

Craig (1985) describe un procedimiento similar, con el cual logró reducir los abandonos en un programa residencial desde el 70 al 20 por ciento. Básicamente, el procedimiento consistía en un programa externo de preparación para el ingreso de una duración de entre 30 y 60 días. Addenbrooke y Rathod (1990) obtuvieron los mismos resultados con alcohólicos y Festinger, Lamb, Kountz, Kirby y Marlowe (1995) con cocainómanos.

Otro ejemplo de la aplicación de este tipo de estrategias se encuentra en el trabajo de Lang (1990), en donde se utilizó lo que el autor denominó "estrategias de rodaje", que consistían en el cumplimiento por parte de los pacientes de determinadas prescripciones antes del tratamiento como condición de entrada en el mismo; por ejemplo: cumplimentar y entregar determinados cuestionarios en un tiempo predeterminado y tomar la medicación de acuerdo con la pauta prescrita. 
La dificultad que plantean estas "estrategias de rodaje" es que, obviamente, ingresan menos sujetos en los programas de tratamiento, auque los que lo hacen tienen mayor probabilidad de finalizarlo. También ofrecen problemas éticos y legales, sobre todo en los recursos públicos, en donde tal vez no sería posible mantener a un sujeto en una lista de espera de manera artificial. Quizás, la solución vendría por plantearlo, no como una condición para ser atendido, sino como una primera fase importante del programa de tratamiento. Es decir, se deberían planificar programas de tratamiento en donde se incluyesen de forma rutinaria este tipo de procedimientos.

\section{b) Anticipar la heterogeneidad. Adecuar los progra- mas a las condiciones de los sujetos}

Se trataría de anticipar las necesidades de los pacientes para así prever las dificultades y acomodar las condiciones del programa a las necesidades de éstos. Por ejemplo, en los programas en donde se atienden a mujeres, una estrategia que se ha mostrado eficaz para incrementar la tasa de retención en los programas residenciales es permitir que los hijos de aquellas que los tengan residan con ellas durante su estancia en el programa, en el cual se incluyen también otros servicios de apoyo, como por ejemplo, cuidados para los niños (Hughes et al., 1995; Szuster, Rich, Chung y Bisconer, 1996; Howell, Heiser y Harrington, 1999).

Pero, el mejor ejemplo de este tipo de estrategias es el diseño de intervenciones específicas para el tratamiento de la drogodependencia de sujetos con psicopatología asociada. En el texto de Solomon, Zimberg y Shollar (1993/1996) se dedican varios capítulos a la exposición de programas de tratamiento específicos de los pacientes con diagnóstico dual.

\section{c) Implicar en el tratamiento a las personas cerca- nas al paciente}

La implicación de personas cercanas al paciente en el tratamiento se ha mostrado como un procedimiento beneficioso en los tratamientos de abuso de drogas (entre otros, Sorenson et al., 1985; De Leon, 1991; Higgins et al., 1994a; Conner et al., 1998).

Por ejemplo, Sisson y Azrin (1993) examinaron el efecto de la aplicación de la Community Reinforcement Approach, CRA (Aproximación de Reforzamiento Comunitario) con y sin participación de la familia. El programa en el que se había incluido el apoyo familiar tuvo muchos menos abandonos que el CRA sin la participación de la familia.

Este procedimiento también se ha mostrado eficaz en otro tipo de programas. Por ejemplo, en un trabajo de Stanton, Steier, Cook y Todd (1984), 164 heroinómanos se asignaron aleatoriamente a dos condiciones de tratamiento: metadona/metadona más participación de la familia. Los resultados mostraron que el segundo grupo tenía muchos menos abandonos que el primero.

\section{d) Terapias de incentivo (manejo de contingencias)}

En este caso, se utiliza el manejo de contingencias para mejorar las tasas de retención. Habitualmente se emplean gratificaciones económicas y, sobre todo, privilegios o actividades gratificantes contingentemente a los resultados negativos en las muestras de orina de detección de consumo de drogas.

Los trabajos del grupo de Higgins con adictos a la cocaína son una buena referencia de este tipo de intervenciones (Higgins et al., 1991, 1993, 1994b) (Tabla 4). En dos de estos trabajos (Higgins et al., 1991, 1993) se comparó un programa multicomponente conductual con un programa de counseling tradicional. En ambos estudios, los sujetos se distribuyeron aleatoriamente a los dos tratamientos. El tratamiento conductual combinaba el Community Reinforcement Program (CRA) con el manejo de contingencias, que consistía en la obtención de vales que canjeaban por determinados privilegios asociados a resultados negativos en las muestras de orina para la detección de cocaína. La terapia de consejo consistía en sesiones de apoyo individuales y grupales y grupos de autoayuda. En ambos estudios la retención fue significativamente mayor en el grupo conductual. En el primer trabajo, el $85 \%$ de los sujetos asignados a este grupo completaron las doce semanas del tratamiento frente al $42 \%$ de los asignados al grupo de counseling. En el segundo trabajo, el 58\% $(11 / 19)$ de los sujetos del grupo conductual completaron las 24 semanas del programa frente al 11\% (2/19) del grupo de counseling. De especial interés es un tercer estudio en el que se llevó a cabo un diseño de desmantelamiento de un programa multicomponente conductual (CRA más manejo de contingencias) con el objetivo de identificar los componentes activos de éste (Higgins et al., 1994b). Cuarenta pacientes fueron asignados al azar al tratamiento conductual de 24 semanas de duración, con o sin el programa de incentivo. La terapia de incentivo se mantuvo durante las doce primeras semanas del programa, mientras que en las semanas 13 a la 24 los dos grupos fueron similares. Los resultados mostraron que el $75 \%$ de los pacientes asignados al grupo de incentivo finalizaron el tratamiento frente al $40 \%$ del programa sin incentivo.

Los resultados de un reciente trabajo de similares características mostraron que el manejo de contingencias mejoraba significativamente la retención, esta vez en un tratamiento ambulatorio con naltrexona (Preston et al., 1999).

Una versión diferente de la utilización del manejo de contingencias para incrementar la adhesión al tratamiento la aportan Brooner, Kidorf, King y Bigelow (1997). Estos autores utilizan la asistencia a la terapia individual como condición previa de la terapia farmacológica. Es decir, la metadona se empleaba como 
Tabla 4. Efecto de las "terapias de incentivo" sobre la retención

\begin{tabular}{llccc}
\hline Autores & Grupos de comparación & $\mathbf{N}^{\mathbf{0}}$ de Sujetos & Duración & $\begin{array}{c}\text { Tasa de retención (\%) } \\
\text { (finalización) }\end{array}$ \\
\hline Higgins et al. (1991) & - Conductual & 13 & 12 semanas & 85 \\
& - Consejo & 15 & & 42 \\
Higgins et al. (1993) & - Conductual & 19 & 24 semanas & 58 \\
& - Consejo & 19 & & 11 \\
Higgins et al. (1994b)) & - Conductual con incentivo & 20 & 24 semanas & 75 \\
& - Conductual sin incentivo & 20 & & 40 \\
\hline
\end{tabular}

estímulo reforzador de la asistencia a la terapia. El seguimiento a largo plazo muestra que los sujetos del grupo experimental obtienen mejores resultados que los del grupo control.

De todos modos, en nuestra opinión resulta bastante contraproducente utilizar precisamente una sustancia adictiva, en este caso un opiáceo, como estímulo reforzador de cualquier conducta en este tipo de pacientes.

\section{e) Combinar terapias farmacológicas con psicológi-} cas

Los tratamientos farmacológicos obtienen mejores tasas de retención que los tratamientos psicológicos. Esta conclusión queda muy bien expuesta en el reciente trabajo de D'Ippoliti, Davoli, Perucci, Pasqualini y Bargali (1998) con 1503 heroinómanos, en el cual se compararon las tasas de retención de tres programas de tratamiento: metadona, programa libre de drogas y naltrexona. Las tasas de retención después de 1 año fueron: $40 \%$ para los pacientes con metadona, $18 \%$ en el programa de naltrexona y $15 \%$ en el programa libre de drogas.

Por otra parte, las terapias psicológicas parecen obtener mayores tasas de retención si se aplican junto a un tratamiento médico. En un trabajo muy bien controlado con 122 adictos a la cocaína y al alcohol, Carroll, Nich, Ball, McCance y Rounsaville (1998) compararon el efecto de dos tratamientos diferentes (cognitivo-conductual y "12 pasos") con y sin apoyo farmacológico (en este caso, disulfiram). En ambos tratamientos, el uso de medicación estuvo asociado con mejoras significativas en las tasas de retención.

Una vez más, se destaca la necesidad de llevar a cabo, si el caso lo requiere, intervenciones combinadas, con el fin de incrementar las tasas de retención y, en definitiva, la eficacia de los programas.

\section{(3) Estrategias relacionadas con los terapeutas}

Se han descrito algunos procedimientos para seleccionar las condiciones de los terapeutas que incrementan los porcentajes de retención. En el traba- jo de McCaul y Svikis (1991) se describe muy bien la aplicación y los resultados de estas estrategias en varios contextos diferentes. Básicamente, se han ensayado tres procedimientos:

a) Incluir el entrenamiento en procedimientos de retención como una parte importante en la formación de los clínicos

Por ejemplo, proporcionar a los clínicos herramientas para prever la heterogeneidad de los sujetos, es decir, ayudar a los terapeutas a desarrollar estrategias para retener a los pacientes con peculiaridades diferentes, respecto a la severidad de la adicción, psicopatología asociada y otras características.

b) Dar incentivos a los terapeutas por incrementar la retención

Entre otros, incentivos económicos, horas libres, flexibilidad en el horario de trabajo o acceso a cursos gratuitos de formación.

c) Procurar la estabilidad y la continuidad de los terapeutas en los programas de tratamiento

La estabilidad y continuidad de los terapeutas a lo largo de las distintas fases del tratamiento es un factor que contribuye a reducir las tasas de abandono de los pacientes. Este hecho a sido bien comprobado en varios estudios. Por ejemplo, en el trabajo ya mencionado de McCaul y Svikis (1991) se encontró que la continuidad del mismo terapeuta en la segunda fase de un tratamiento, que había sido precedida por una fase residencial, era un importante predictor de la retención.

\section{CONCLUSIONES}

La retención de los pacientes en el tratamiento es un fenómeno complejo y, por tanto, se debe ser muy cautos a la hora de ofrecer conclusiones sobre los factores que afectan al abandono. Se hace difícil establecer generalizaciones válidas debido a la diversidad de 
las variables estudiadas y de los criterios para definir tales variables, a la variedad de los procedimientos empleados, y la heterogeneidad de los pacientes y de los programas incluidos en los estudios.

No obstante, parece bastante evidente que los factores relacionados con el abandono tienen que ver con una combinación de las características individuales y situacionales, con especial énfasis en los procesos o parámetros del tratamiento. Es decir, la retención y el abandono son el resultado de una interacción compleja entre determinadas características de los pacientes y los parámetros del tratamiento.

Es posible que, dependiendo del tipo de programa, una misma variable pueda ejercer efectos diferentes sobre el cumplimiento del programa por parte de los pacientes. Por ejemplo, la variable "poseer un buen soporte familiar" (especialmente durante las horas que el paciente pasa fuera del centro), podría ser indicadora de permanencia en un centro de día, y no ejercer un papel similar en otro tipo de programa.

En resumen, no hay una variable clave de la que dependa el abandono o la continuación en la terapia. De hecho, las investigaciones apelan a diferentes factores que, en conjunto, pueden llegar a discriminar al sujeto que abandona del que permanece en tratamiento. Es evidente la existencia de una multideterminación en el proceso de abandono, cosa que, por otra parte, concuerda con la multiplicidad de factores que subyacen en la adquisición y mantenimiento del hábito adictivo.

Una conclusión importante que se podría sacar de los estudios revisados es que las altas tasas de abandono que habitualmente se observan en los programas de drogodependencias no son inevitables.

Diversos estudios empíricos han demostrado que existen procedimientos eficaces para reducir las tasas de abandono, tanto en los tratamientos psicológicos o médicos en general, como en los programas de abuso de drogas en particular. Estos procedimientos tienen que ver con diferentes manipulaciones relacionadas con determinadas características de los pacientes, con los parámetros del tratamiento y con las condiciones en que los clínicos realizan su trabajo.

Algunas de las estrategias que se acaban de comentar tienen suficiente respaldo empírico y su eficacia ha sido verificada (por ejemplo, la combinación de terapias farmacológicas con intervenciones psicoeducativas), mientras que otras necesitan aún de una comprobación práctica más rigurosa. Por tanto, sería recomendable la elaboración de trabajos bien controlados que estudiasen y perfeccionasen estas estrategias.

Por último, los resultados de los estudios revisados ofrecen la evidencia de que, en general, el incremento de las tasas de retención de los programas está asociado con el incremento de la abstinencia a las dro- gas y la mejora en otras variables (conductas delictivas, situación laboral, etc.) que suelen estar asociadas a este tipo de problemas.

\section{REFERENCIAS BIBLIOGRÁFICAS}

Addenbrooke, W.M. y Rathod, N.H. (1990). Relationship between waiting time and retention in treatment amongst substance abusers. Drug and Alcohol Depende, 26, 255-264.

Aron, W.S. y Daily, D.W. (1976). Graduates and splitees from therapeutic community drug teratment programs: A comparison. International Journal of the Addictions, 11, $1-18$

Aszalos, R., McDuff, D.R., Weintraub, E., Montoya, I. y Schwartz, R. (1999). Engaging hospitalized heroin-dependent patients into substance abuse treatment. Journal of Substance Abuse Treatment, 17, 149-158.

Baekeland, F. y Lundwall, L. (1975). Dropping out of treatment: A critical review. Psychological Bulletin, 5, 738783.

Berger, H. y Smith, M.J. (1978). Voluntary versus prescribed termination of methadone maintenance. British Journal of Addiction, 73, 178-180.

Bien, T.H., Miller, W.R. y Tonigan, J.S. (1993). Brief interventions for alcohol problems: A review. Addiction, 88, 305-325.

Brooner, R.K., Kidorf, M., King, V.L. y Bigelow, G.E. (1997). Using behaviorally contingent pharmacotherapy in opioid abusers enhances treatment outcome. En L.S. Harris (Ed.), Problems of drug dependence 1996 (NIDA Research Monograph, 174). Washington, DC: US Deparment of Health and Human Services.

Carroll, K.M. (1997). Enhancing retention in clinical trials of psychosocial treatments: Practical strategies. En L.S. Onken, J.D. Blaine, y J.J. Boren (Eds.), Beyond therapeutic alliance: Keeping the drug-dependent individual in treament (NIDA Research Monograph, $n^{\circ}$ 165. Rockville, MD: US Department of Health and Human Services.

Carroll, K.M., Nich, C., Ball, S.A., McCance, E. y Rounsavile, B.J. (1998). Treatment of cocaine and alcohol dependence with psychotherapy and disulfiram. Addiction, 93, 713-727.

Conner, K.R., Shea, R.R., McDermott, M.P., Grolling, R., Tocco, R.V. y Baciewicz, G. (1998). The role of multifamily therapy in promoting retention in treatment of alcohol and cocaine dependence. American Journal of Adicctions, 7, 61-73.

Craig, R.J. (1985). Reducing the treatment drop out rate in drug abuse programs. Journal of Substance Abuse Treatment, 2, 209-219.

Craig, R.J. y Olson, R.E. (1988). Differences in psychological need hierarchy between program completers and dropouts from a drug abuse treatment program. American Journal of Drug and Alcohol Abuse, 1, 89-96. 
De Leon, G. (1991). Retention in drug-free therapeutic communities. En R.W. Pickens, C.G. Leukefeld y C.R. Schuster (Eds.), Improving drug abuse treatment. NIDA Research Monograph Series, $n^{\circ} 106$. Rockville, MD: US Department of Health and Human Services.

De Leon, G. y Jainchill, N. (1986). Circumstances, motivation, readiness and suitability (CMRS) as correlates of treatment tenure. Journal of Psychoactive Drugs, 8 , 203-208.

De Leon, G., Melnick, G. y Kressel, D. (1997). Motivation and readiness for therapeutic community treatment among cocaine and other drug abusers. American Journal of Drug and Alcohol Abuse, 23, 169-189.

De Leon, G., Melnick, G., Kressel, D. y Jainchill, N. (1994). Circumstances, motivation, readiness, and suitability (the CMRS) scales: Predicting retention in therapeutic community treatment. American Journal of Drug and Alcohol Abuse, 20, 495-515.

De Leon, G. y Schwartz, S. (1984). Therapeutic Communities: What are the retention rates. American Journal of Drug Abuse, 10, 267-284.

Del Rio, M., Mino, A. y Perneger, T.V. (1997). Predictors of patient retention in a newly established methadone maintenance treatment programme. Addiction, 92, 1353-1360.

Des Jarlais, D.C., Joseph, H. y Schmeidler, J. (1983). Predicting postreatment narcotic use among patients termination from methadone maintenance. Harwoth Press.

D'Ippoliti, D., Davoli, M., Perucci, C.A., Pasqualini, F. y Bargagli, A.M. (1998). Retention in treatment of heroin users in Italy: The role of treatment type and of methadone maintenance dosage. Drug and Alcohol Dependence, 52, 167-171.

Erickson, J.R., Stevens, S., McKnight, P. y Figueredo, A.J. (1995). Willingness for treatment as a predictor of retention and outcomes. Journal of Addictive Diseases, 14, 135-150.

Festinger, D.S., Lamb, R.J., Kountz, M.R., Kirby, K.C. y Marlowe, D. (1995). Pretreatment dropout as a function of treatment delay and client variables. Addictive Behaviors, 20, 111-115.

Garfield, S.L. (1994). Research on client variables in psychotherapy. En A.E. Bergin y S.L. Garfield (Ed.), Handbook of psychotherapy and behavior change. New York: John Wiley \& Sons.

González-Saiz, F., De Leon, G., Macías, C.G., Lillo, P., Varela, L., Castro, E., Gómez, E., Ruiz, N., Montañés, M. y Salvador-Carulla, L. (1998). La motivación y las actitudes ante el tratamiento como predictores de abandono precoz en Comunidad Terapéutica: datos preliminares de la adaptación de la escala CMRS. V Encuentro Nacional sobre drogodependencias y su Enfoque Comunitario. Cádiz.

Gossop, M. (1978). Drug dependence: A study of the relationship between motivational, cognitive, social and historical factors, and treatment variables. The Journal of Nervous and Mental Disease, 166, 44-50.
Gutiérrez, M., Ballesteros, J., González-Oliveros, R. y Ruiz de Apodaka, J. (1995). Tasa de retención en dos programas de naltrexona para heroinodependientes en Vitoria, España. Revista de Psiquiatría de la Facultad de Medicina de Barcelona, 22, 215-225.

Higgins, S.T., Budney, A.J., Bickel, W.K. y Badger, G.J. (1994a). Participation of significant others in outpatient behavioral treatment predicts greater cocaine abstinence. American Journal of Drug and Alcohol Abuse, 20, 47-56.

Higgins, S.T., Budney, A.J., Bickel, W.K., Foerg, F.E., Donham, R. y Badger, G.J. (1994b). Incentives improve outcome in outpatient behavioral treatment of cocaine dependence. Archives of General Psychiatry, 51, 568576.

Higgins, S.T., Budney, A.J., Bickel, W.K., Hughes, J.R., Foerg, F. y Badger, G. (1993). Achieving cocaine abstinence with a behavioral approach. American Journal of Psychiatry, 150, 763-769.

Higgins, S.T., Delaney, D.D., Budney, A.J., Bickel, W.K., Hughes, J.R., Foerg, F. y Fenwick, J.W. (1991). A behavioral approach to achieving cocaine abstinence. American Journal of Psychiatry, 148, 1218-1224.

Howell, E.M., Heiser, N. y Harrington, M. (1999). A review of recent findings on substance abuse treatment for pregnant women. Journal of Substance Abuse Treatment, 16, 195-219.

Hubbard, R.L., Rachal, J.V., Craddok, S.G.Y. y Cavanaugh, E.R. (1984). Treatment outcome prospective Study (TOPS): Client characteristics and behaviors before, during and after treatment. En J.F. Tims y J.P. Ludorf (Eds.), Drug abuse treatment evaluation: strategies progress and prospects. Maryland: NIDA.

Hughes, P.H., Coletti, S.D., Neri, R.L., Urmann, C.F., Stahl, S., Sicilian, D.M. y Anthony, J.C. (1995). Retaining cocaineabusing women in a therapeutic community: The effect of a child live-in program. American Journal of Public Health, 85, 1149-1152.

Joe, G.W., Simpson, D.D. y Broome, K.M. (1998). Effects of readiness for drug abuse treatment on client retention and assessment of process. Addiction, 93, 1177-90.

Kooyman, M. (1993/1996). La Comunidad Terapéutica para Drogodependientes. Bilbao. Mensajero.

Lang, J.M. (1990). The use of a run-in to enhance compliance. Statistics in Medicine, 9, 87-95.

Machado, M.J. y Girón, S. (1993). Perfil psicosocial y retención de heroinómanos en tratamiento ambulatorio. Adicciones, 2, 109-125.

MacNair, R.R. y Corazzini, J.G. (1994). Client factors influencing group therapy dropouts. Psychotherapy, 31, 352362.

McCaul, M.E. y Svikis, D.S. (1991). Improving client compliance in outpatient treatment: counselor-targeted interventions. En R.W. Pickens, C.G. Leukefeld y C.R. Schuster (Eds.), Improving drug abuse treatment. NIDA Research Monograph Series, $n^{\circ}$ 106. Rockville, MD: US Department of Health and Human Services. 
Melnick, G., De Leon, G., Hawke, J., Jainchill, N. y Kressel, D. (1997). American Journal of Drug and Alcohol Abuse, 23, 485-506.

Miller, R.R. y Rollnick, S. (1991). Motivational Interviewing. Preparing people to change addictive behavior. New York: The Guilford Press.

Ochoa, E., Arias, F., Somoza, J.C., Vicente, N. Y Jordá, L. (1992). Retención en un programa de tratamiento con naltrexona: dos años de seguimiento. Adicciones, 4, 207-212.

O'Malley, J.E., Anderson, W.H. y Lazare, A. (1972). Failure of outpatient treatment of drug abuse: I. Heroin. American Journal of Psychiatry, 128, 865-868.

Preston K.L., Silverman K., Umbricht A., De Jesus A., Montoya I.D. y Schuster, C.R. (1999). Improvement in naltrexone treatment compliance with contingency management. Drug and Alcohol Dependence, 54, 127-135.

Prochaska, J.O. y DiClemente, C.C. (1986). Toward a comprehensive model of change. En W.R. Miller y N. Heather (Eds.), Treating addictive behaviors: Processes of change. New York: Plenum Press.

Prochaska, J.O., DiClemente, C.C. y Norcross J.C. (1992). In search of how people change. Applications to addictive behaviors. American Psychologist, 47, 1102-1114.

Sánchez-Carbonell, J., Camí, J. y Brigos, B. (1988). Follow-up of heroin addicts in Spain (EMETYST project): Results 1 year after treatment admision. British Journal of addiction, 83, 1439-1449.

Sánchez, L. y Matellanes, M. (1993). La especialización de los recursos para la atención de las drogodependencias. Adicciones, 2, 127-140.

Saunders, B., Wilkinson, C. y Allsop, S. (1991). Motivational intervention with heroin users attending a methadone clinic. En W.R. Miller y S. Rollnick (Eds.), Motivational Interviewing: Preparing People to Change Addictive Behavior. New York: Guilford.

Secades Villa, R., Fernández Rodríguez, R. y Fernández Hermida, J.R. (1998). Factores asociados a la retención de heroinómanos en un programa libre de drogas. Adicciones, 10, 53-58.

Secades Villa, R. y Menéndez Cuartas, A. (1997). Factores que determinan la retención de heroinómanos en un centro de día. Revista Española de Drogodependencias, 22, 5-12.

Simpson, D.D. (1984). National treatment system evaluation based on the Drug Abuse Reporting Program (DARP) followup research. En F.M. Tims y J.P. Ludford (Eds.), Drug abuse treatment evaluation: Strategies, progress, and prospects. NIDA Research Monograph, $n^{\circ}$ 51. Washington, DC:US Deparment of Health and Human Services.

Simpson, D.D., Joe, G.W. y Rowan-Szal, G.A. (1997). Drug abuse treatment retention and process effects on follow-up outcomes. Drug and Alcohol Dependence, 47, 227-235.

Sirotnik, K.A. y Roffe, M.W. (1978). An investigation of the feasibility of predicting outcome indices in the treatment of heroin addiction. International Journal of the Addictions, 12, 755-775.

Sisson, R.W. y Azrin, N.H. (1993). Family-member involvement to initiate and promote treatment of problem drinkers. Journal of Behabior Therapy and Experimental Research, 17, 15-21.

Solomon, J., Zimberg, S. y Shollar, E. (1993/1996). Diagnóstico dual. Barcelona: Ediciones en Neurociencias.

Sorenson, J.L., Gibson, D., Bernal, G. y Deitch, D. (1985). Methadone applicant dropouts: Impact of requiring involvement of friends and family in treatment. International Journal of the Addictions, 20, 1273-1280.

Stanton, M.D., Steier, F., Cook, L. y Tood, T.D. (1984). Narcotic detoxification in a family and home contest: Final report-1980-1983. Rockville, MD: National Institute on Drug Abuse.

Stark, M.J. (1992). Dropping out of substance abuse treatment: A clinically oriented review. Clinical Psychology Review, 12, 93-116.

Stark, M.J., Campbell, B.K. y Brinkerhoff, C.V. (1990). “Hello, May we help you?" A study of attrition prevention at the time of the first phone contact with substance-abusing clients. American Journal of Drug and Alcohol Abuse, 16, 67-76.

Swett, C. y Noones, J. (1989). Factors associated with premature termination from outpatients treatment. Hospital and Community Psychiatry, 40, 947-951.

Szuster, R.R., Rich, L.L., Chung, A. y Bisconer, S.W. (1996). Treatment retention in women's residential chemical dependency treatment: The effect of admission with children. Substance Use and Misused, 31, 1001-1013.

Tucker, J.A., Vuchinich, R.E. y Harris, C.V. (1985). Determinants of substance abuse relapse. En M. Galizio y S.A. Maisto (Eds.), Determinants of substance abuse: Biological, psychological and environmental factors. New York: Plenum Press.

Vaillant,G.E. (1966). A 12-year follow-up of New York narcotic addicts III. Archives of General Psychiatry, 15, 599-609.

Wells, E.A., Peterson, P.L. y Gaine, R.R. (1994). Outpatient treatment for cocaine abuse: A controlled comparison of relapse prevention and twelve-step approaches. American Journal of Drug and Alcohol Abuse, 20, 1-17.

Wexler, H.K. y De Leon, G. (1977). The Therapeutic Community: Multivariate Prediction of Retention. American Journal of Drug and Alcohol Abuse, 4, 145-151.

Wierzbicki, M. y Pekarik, G.A. (1993). A meta-analysis of psychotherapy dropout. Professional Psychology, Research and Practice, 24, 190-195. 
